\title{
Simulations and Virtual Learning Supporting \\ Clinical Education During the COVID 19 Pandemic [Response To Letter]
}

This article was published in the following Dove Press journal:

Advances in Medical Education and Practice

\section{Shima Tabatabai $(\mathbb{D}$}

Medical Ethics and Law Research Center, Shahid Beheshti University of Medical

Sciences, Tehran, Iran
Correspondence: Shima Tabatabai

Medical Ethics and Law Research Center,

Shahid Beheshti University of Medical

Sciences, Tehran, Iran

Tel +982I 224056 I I

Email shtabatabai@yahoo.com

\section{Dear editor}

I would like to appreciate the opportunity to respond to the issues raised in Novintan et al. As a Post-Doctoral researcher in the filed of medical education, I would like to clarify some futuristic aspects of my commentary. ${ }^{1}$ I would also like to thank Dr. Novintan and colleagues, the final year medical students at Imperial College School of Medicine for their interest in my article and for taking the time to express their concerns about the virtual platforms' ability to meet the practical needs of students. ${ }^{2}$

I acknowledge the importance of using virtual reality, simulated and virtual patients (computer-based simulations of patients) for both training and assessment in medical education. Novintan noted potential concerns with the comprehensiveness of the virtual objective structured clinical exam (OSCE) to provide a platform to address the clinical education needs. I agree that there are notable differences between OSCEs and VOSCEs, as well as in many other clinical competency exams. I also agree that virtual reality ward rounds provide a live-stream of the consultantpatient interaction on the ward, allowing isolated students to take histories from patients and prepare students for clinical scenarios and exams.

The OSCE is one of the most valid, reliable tests for the assessment of clinical skills. The OSCE is that structured type of exams- trying to be more objective than the merely passive observation during clinical rotations and that they assess clinically relevant procedures like patient interviews, medical decision making, and practical tasks.In a typical OSCE, examinees rotate through several stations staffed by either real or standardized patients, and stations equipped with mannequins or plastic models where they are required to perform different clinical tasks. Simulated patients could be useful as assessment tools in OSCEs or in other assessment methods in evaluating students' interactions with patient-related medical issues, such as problem-solving and clinical reasoning abilities.

The discussion in my paper approached from the futuristic perspective of a medical educationalist. Simulation is increasingly becoming a cornerstone of clinical training. ${ }^{1}$ Currently, technology-based simulation using in educational programs classified into augmented and virtual reality. Augmented reality integrates three-dimensional virtual objects into the user's physical environment in real-time. Virtual reality (VR) is emerging as a new method of delivering simulation. VR 
simulates the user's physical existence by providing multisensory, three-dimensional environments that enable them to become fully immersed in a simulated world. ${ }^{3}$

VR simulation is used in medical and nursing schools as well as in postgraduate education around the world, and offers benefits for learners and educators, delivering safe, standardized, repeatable, cost-effective clinical training on demand.

Novintan et al stated, at present, available technologies cannot replicate all of the practical skills and cannot replicate in the virtual assessment. I agree that Technologies still need to progress before VR becomes a comprehensive assessment platform. Despite this, the future is promising. The Advancements in VR technology make its use as a training and assessment tool in OSCEs possible and there are advantages over current methods.

In Taiwan, a health care training program using virtual reality (VR) in performing the Port-A cath technique and administration of medications for novice nurses has led them to practice and perform more invasive skills. Accordingly, nurses must be well-prepared before teaching by their mentors. One study revealed that VR simulation significantly reduced error rates. The nurses who participated in the VR simulation may better prepare for inserting the Port-A cath. Moreover, the knowledge score regarding the Port-A cath technique was significantly higher in the nurses that participated in the simulation training than in the control group. ${ }^{4}$

VR simulations also have been used to implement a clinical competency assessment. Recently, for supporting active and reflective learning, the virtual patients (VP) have been used as a complementary method to simulated patients. ${ }^{5}$ However, the fact that interactive VR involves an immersive, dynamic, adaptive, interactive world should be concerned in online OSCEs and Virtual OSCEs (VOSCEs). In the context of clinical training, this can include virtual stations, interactive standardized VP, colleagues, and relatives, with interaction similar to the real world. For example, in a scenario of a VP with chest pain in an emergency department, the learner could move in the virtual environment and interact with VP as they would in a real situation. They can take a history, examine, investigate, diagnose, and treat the patient.

Interactive simulation of patients (ISP) is a comprehensive and high-fidelity virtual patient-based learning tool. ISP has been designed for that purpose to resemble a real patient encounter covering extensive functions for medical history, physiological examination procedures, and laboratory/imaging tests. The system aims to help medical and healthcare students to practice clinical reasoning and practical skills. $^{5}$

Virtual Patient (VP) cases with automated scoring could be used as a complementary method for both formative and summative assessment. Before the VP-based systems and computer-based simulations like ISP can be used in exams, Students need to be trained for in mastering the assessment tools. If such systems would be used in high-stake exams, the scoring should be validated automatically. ${ }^{6}$ Strength of ISP-like systems is that these VR cases can score automatically and immediately present results of the examination thus reducing the costs. A study indicates that ISP is able to present and simulate realistic patient encounters to an acceptable level of complexity and allow differentiation of one student's performance from another, including gender-related differences. ${ }^{6}$

In my commentary article, I discussed the idea of utilizing VR for OSCEs and emphasized the benefits of integrating this technology in clinical assessment.

The idea of virtual reality simulations specifically designed for OSCEs is not a new concept, however. Few studies explored the applicability of VR for OSCEs in the assessment of medical students. A study published in 2008 first demonstrated the efficacy of using computerized VP as a tool for testing clinical reasoning and problem-solving amongst students. The results were favorable, with students showing a positive response to the new examination style. ${ }^{6}$

Additionally, multiple studies explored the ability of virtual reality to provide a suitable means of teaching communication skills and to assess students' responses to a scenario involving a VP demanding antibiotics. The VR potential to simulate such scenarios suggests that its use for a communication-based OSCE station is applicable.,

Researchers explore the use of virtual reality to simulate the practical element of clinical skill procedures in the field of dental education and The potential benefits of two computerized VR dental simulators. These simulators help dental students to enhance their skills on computer-generated phantom heads rather than using the actual mechanical model. ${ }^{7}$ At the end of the simulation, an evaluation report and a list of procedural errors can produce for each student as a feedback tool). With the technology already in place for dental education, the jump to developing a VR clinical skill OSCE station is viable.

Based on these pieces of evidence, I disagree with Novintan as a virtual OSCE make the exam more 
incomprehensive and provide an inaccurate score of the student's capabilities, but instead make the OSCE more dynamic, adaptive, interactive, and safe in through the pandemic situation.

Nevertheless, the main challenges of a Virtual OSCE station are whether haptic technology (touch sensation) integrated with VR is suitable and enough to surrogate the actual performance of the clinical procedure on the standard patient, mannequins, or plastic models. ${ }^{5}$ Can VR be enough to simulate practical skills and help medical students to be competent in completing procedures on reallife patients? We should consider that the conventional educational plastic mannequins and models are even less realistic. The VR learning experiences and VP in VOSCE station would be more simulate the real situation and real patients. With the more future advance in technology, VR could be integrated with haptic technology (touch sensation) to produce reality-like clinical scenarios.

\section{Conclusion}

The introduction of the VOSCE into medical school will lead us about the variety of factors that limited the utilization of more objective, standardized methods for examining clinical skills and gave rise to difficulties in the management of changes in assessment methods.

Virtual reality technology within the medical field has already shown incredible results. The future VR and ISP in medical education are truly promising. Of course, there is still a long way to go before VR becomes the platform for OSCEs. However, Advancements in VR simulation-based technology make its use as an assessment tool in OSCEs possible, and the potential for VOSCEs makes a positive impact within the future of medical education and assessment over conventional methods.

\section{Disclosure}

The author reports no conflict of interest in this communication.

\section{References}

1. Tabatabai S. Simulations and virtual learning supporting clinical education during the COVID 19 pandemic. Adv Med Educ Pract. 2020;11:513-516. doi:10.2147/AMEP.S257750

2. Novintan S, Mann S, Hazemi-Jebelli Y. Simulations and virtual learning supporting clinical education during the COVID 19 pandemic [Letter]. Adv Med Educ Pract. 2020.

3. Pottle J. Virtual reality and the transformation of medical education. Future Healthc J. 2019;6(3):181-185. doi:10.7861/fhj.2019-0036

4. Tsai S, Chai S, Hsieh L, et al. The use of virtual reality computer simulation in learning port-A cath injection. Adv in Health Sci Educ. 2008;13:71-87. doi:10.1007/s10459-006-9025-3

5. Thakker A, Devani P. Is there a role for virtual reality in objective structured clinical examinations (OSCEs)? MedEdPublish. 2019;8(3). doi:10.15694/mep.2019.000180.1

6. Courteille O, Bergin R, Stockeld D, et al. The use of a virtual patient case in an OSCE-based exam-a pilot study. Med Teach. 2008;30(3): e66-76. doi:10.1080/01421590801910216

7. Huang TK, Yang CH, Hsieh YH, et al. Augmented reality (AR) and virtual reality (VR) applied in dentistry. Kaohsiung J Med Sci. 2018;34 (4):243-248. doi:10.1016/j.kjms.2018.01.009

\section{Publish your work in this journal}

Advances in Medical Education and Practice is an international, peerreviewed, open access journal that aims to present and publish research on Medical Education covering medical, dental, nursing and allied health care professional education. The journal covers undergraduate education, postgraduate training and continuing medical education including emerging trends and innovative models linking education, research, and health care services. The manuscript management system is completely online and includes a very quick and fair peer-review system. Visit http://www.dovepress.com/testimonials.php to read real quotes from published authors. 